\title{
Triggering of a volcanic dome collapse by rainwater infiltration
}

\author{
Peter D. Hicks, ${ }^{1,2}$ Adrian J. Matthews, ${ }^{3}$ and Mark J. Cooker ${ }^{1}$ \\ Received 30 July 2009; revised 3 February 2010; accepted 19 April 2010; published 30 September 2010.
}

[1] The thermodynamic processes in a one-dimensional model of a porous lava dome are considered in the presence of a rising magmatic gas flux through the void spaces and rainfall interacting with the dome surface. The steady state surface temperature of the dome depends on both magmatic gas mass flux and rainfall rate. A critical rainfall rate is determined, that cools the dome surface to $100^{\circ} \mathrm{C}$. Rainfall rates above this critical value allow liquid infiltration into the void spaces of the dome, thus restricting the escape of magmatic gas. A model which restricts the gas flow through the surface predicts internal gas pressures much higher than the overburden pressure in the top few meters, approximately one hour after the onset of rainfall. For a marginally stable dome, this could cause small Vulcanian explosions, which (depending on their location) could trigger a dome collapse, on a timescale consistent with observations.

Citation: Hicks, P. D., A. J. Matthews, and M. J. Cooker (2010), Triggering of a volcanic dome collapse by rainwater infiltration, J. Geophys. Res., 115, B09212, doi:10.1029/2009JB006831.

\section{Introduction}

[2] The triggering of volcanic activity by external factors has received much attention [e.g., Neuberg, 2000]. Rainfall has been shown to trigger activity at several volcanoes, including Mount St Helens, U.S.A. [Mastin, 1994], Unzen, Japan [Yamasato et al., 1998], Merapi, Indonesia [Voight et al., 2000], Piton de la Fournaise, Réunion island [Violette et al., 2001], the Soufrière Hills Volcano (SHV), Montserrat [Matthews et al., 2002; Carn et al., 2004; Barclay et al., 2006; Matthews et al., 2009], and Stromboli, Italy [Hort et al., 2003]. At lava dome forming eruptions, rainfall triggered activity includes lava dome collapses, explosions and pyroclastic flows.

[3] Several theories have been suggested to explain this activity: infiltration of water into a volcanic dome [Elsworth et al., 2004; Matthews and Barclay, 2004], cooling of a dome by rainfall causing thermal contraction and associated internal stresses [Mastin, 1994; Yamasato et al., 1998], and the action of pressurized water or steam on pre-existing internal failure surfaces in the dome [Elsworth et al., 2004; Simmons et al., 2004; Taron et al., 2007]. In these models, the volcanic system is assumed to be marginally stable, and rainfall is the last nudge toward instability. However, a question remains: how does rainwater on the surface of the dome infiltrate the dome interior?

[4] Recently, Hicks et al. [2009] modeled the surface layers of a volcanic dome as a one-dimensional porous

\footnotetext{
${ }^{1}$ School of Mathematics, University of East Anglia, Norwich, UK.

${ }^{2}$ Now at Department of Mathematics, University College London, London, UK.

${ }^{3}$ School of Environmental Sciences and School of Mathematics, University of East Anglia, Norwich, UK.

Copyright 2010 by the American Geophysical Union. 0148-0227/10/2009JB006831
}

matrix through which magmatic gas rises from a deep source. When surface energy fluxes due to radiative and convective (sensible) heat loss were included, the model predicted theoretical steady state internal temperature profiles that were consistent with observations. The main controlling parameter was the imposed flux of magmatic gas at the lower boundary. A realistic gas flux of $3.5 \times 10^{-3} \mathrm{~kg} \mathrm{~s}^{-1} \mathrm{~m}^{-2}$ [Edmonds et al., 2001] gave a realistic steady surface temperature of $210^{\circ} \mathrm{C}$ [e.g., Oppenheimer et al., 1993]. Here, we extend the "dry" model of Hicks et al. [2009] by adding rainfall in the form of an additional surface energy flux from rainwater evaporation.

\section{Model Description}

[5] A lava dome is a complex porous medium, with individual features occurring over a wide range of length scales, from individual discrete vesicles of perhaps a millimeter in diameter, through connected vesicle networks, cracks, fissures, fumaroles and faults, the maximum width of which might be several centimeters. The analysis of the evolution of energy and magmatic gas properties is simplified dramatically in regions which are in thermal equilibrium, that is regions in which it is valid to assume the rock matrix and magmatic gas share a common temperature $T(z, t)$. Locally, small variations in magmatic gas flux could give rise to a magmatic gas temperature that is different from the temperature of the porous matrix through which it is flowing. A non-thermal-equilibrium model would include an energy conservation equation for each phase, coupled by (difficult to constrain) heat transfer terms [see, e.g., Kaviany, 1995]. The time-scale over which thermal equilibrium is recovered can be approximated by

$$
t \sim \frac{\ell^{2} \rho_{r} c_{r}}{k_{r}}
$$




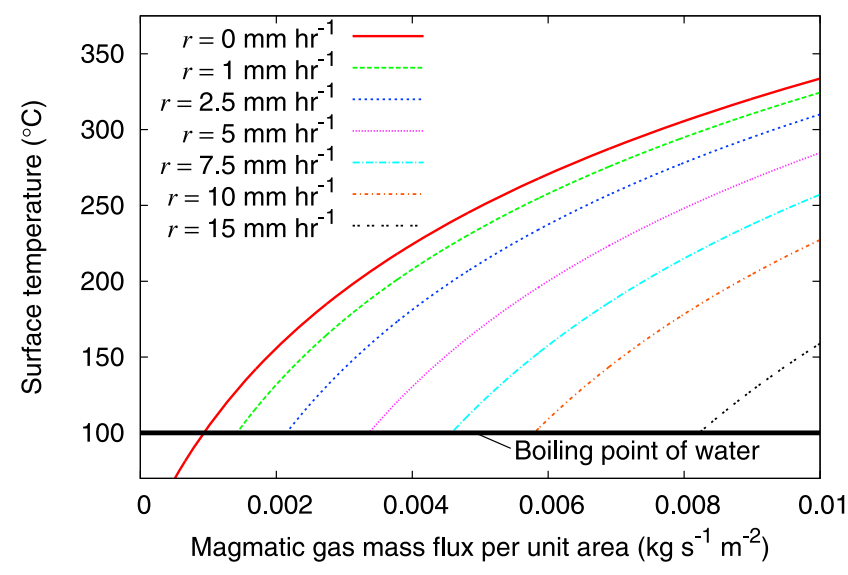

Figure 1. Steady state surface temperature $\left(T_{s}\right)$ as a function of magmatic gas mass flux per unit surface area $\left(m_{g}\right)$ for rainfall rates $r$, between 0 and $15 \mathrm{~mm} \mathrm{hr}^{-1}$.

where $\rho_{r}, c_{r}$ and $k_{r}$ are the density, specific heat capacity and thermal conductivity of the dome matrix, respectively; and $2 \ell$ is a typical length scale separating individual porespaces. The assumption of thermal equilibrium is valid in regions of the dome for which the associated time-scale is much smaller than the time-scale of the process we wish to model. Observations [Carn et al., 2004] and statistical analysis [Matthews et al., 2009] of rainfall induced lava dome activity suggest a delay of the order of 100 minutes between the onset of rainfall and the resulting dome activity. With $t=$ 100 minutes, equation (1) gives a maximum separation distance between adjacent pores of $2 \ell=16 \mathrm{~cm}$, if thermal equilibrium is to hold. This condition is satisfied in the significant portions of the dome, with the exception between cracks in the least porous regions of the dome. However, we shall see that liquid infiltration and the resulting over pressures are greatest in the high porosity regions of the dome and therefore a local thermal equilibrium assumption can account for the energy evolution in the regions of the dome of most interest.

[6] In regions in thermal equilibrium, heat transfer is dominated by diffusion through the matrix and by the upward transport by a mobile magmatic gas, although additionally diffusion within the magmatic gas is also included within the model. With these assumptions the energy conservation equation is given by a time-dependent, parabolic partial differential equation. Within the void-spaces of the porous matrix, the volume flux of magmatic gas is calculated using Darcy's law and is proportional to the magmatic gas pressure gradient. The temperature is related to the magmatic gas pressure $p_{g}(z, t)$, and density $\rho_{g}(z, t)$ through the ideal gas equation. Using Darcy's law and the ideal gas equation, the mass conservation equation is manipulated to give a second time-dependent, parabolic partial differential equation, for the magmatic gas density. Further details of the model derivation are given in the work by Hicks et al. [2009] and Appendix A. Each differential equation of this type requires a boundary condition at the surface and at depth.

[7] The vertical coordinate extends from $z=0$ at the dome surface down to a lower boundary at $z=-L=-20 \mathrm{~m}$, well below the surface thermal boundary layer, so that the model solutions are insensitive to its exact value. Heat is transferred by diffusion through the matrix and by the upward transport of magmatic gas. The temperature $T(z)$, at the lower boundary is held constant at $T_{L}=1100 \mathrm{~K}$, and the magmatic gas mass flux $m_{g}$ is prescribed. At the upper boundary, a (Dirichlet) boundary condition, in which the magmatic gas pressure is set to equal the atmospheric pressure, is used. For the temperature, a (Neumann) boundary condition relates the temperature gradient to the surface heat flux $H$. The surface heat flux is the sum of the radiative and convective fluxes, both of which are functions of the surface temperature, $T_{s}$. In this paper, we add rainwater falling at a rate $r$, which is evaporated from the hot surface, such that

$H\left(T_{s}, r\right)=-\varepsilon \sigma\left(T_{s}^{4}-T_{a}^{4}\right)-S_{h} \rho_{a} c_{a} u_{a}\left(T_{s}-T_{a}\right)-r \rho_{l}\left(c_{l} \Delta T+h_{l g}\right)$,

where, $\epsilon=0.95$ is emissivity, $\sigma$ is the Stefan-Boltzmann constant, $T_{a}=298 \mathrm{~K}$ is the effective atmospheric temperature, $S_{h}=0.002$ is the roughness of the dome surface, $\rho_{a}$, $\rho_{l}$ are the densities, and $c_{a}, c_{l}$ are the specific heat capacities of air and water, respectively, $u_{a}$ is the surface wind speed, $\Delta T=75 \mathrm{~K}$ is the temperature change needed to heat rainwater from the ambient air temperature to boiling point, and $h_{l g}$ is the latent heat of vapourisation of water. Further details can be found in the work by Hicks et al. [2009]. The model extends that of Matthews and Barclay [2004], with the crucial addition of the advection of heat by magmatic gas flow.

\section{Steady State Solutions}

[8] Steady state solutions similar to those in the dry model of Hicks et al. [2009] can be found, modified by the addition of the surface energy flux due to rainfall. The steady state temperature profile is

$$
T(z)=T_{L}-\frac{H\left(T_{s}, r\right)}{m_{g} c_{g}}\left(e^{\beta z}-e^{-\beta L}\right),
$$

where $\beta=m_{g} c_{g} / k_{e}$, with $k_{e}=2.1 \mathrm{~W} \mathrm{~m}^{-1} \mathrm{~K}^{-1}$ the effective thermal conductivity of the dome matrix. The quantity $1 / \beta$ is the thickness scale for the surface thermal boundary layer. The surface temperature $T_{s}$, is the smallest positive root of the quartic equation

$$
T_{s}=T_{L}-\frac{H\left(T_{s}, r\right)}{m_{g} c_{g}}\left(1-e^{-\beta L}\right),
$$

where $H\left(T_{s}, r\right)$ is defined by equation (2). Steady state profiles for magmatic gas pressure and magmatic gas density in a dry dome can also be found [Hicks et al., 2009]. If the magmatic gas mass flux at the lower boundary is increased, more heat is advected to the surface. This increases the surface temperature. The surface heat loss (due to radiative and convective fluxes) then also increases, until a new equilibrium is reached. Hence, the steady state surface temperature increases with increasing magmatic gas flux, as shown by the thick line in Figure 1, for zero rainfall rate. When rainfall is included, the extra cooling heat flux from surface evaporation decreases the steady state surface temperature (Figure 1). 


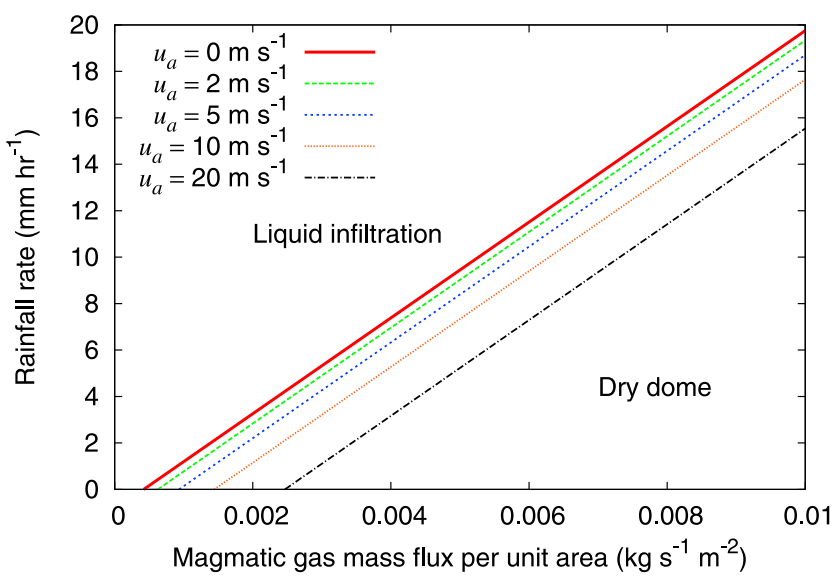

Figure 2. Regime diagram showing the critical rainfall rate $\left(r_{c}\right)$ to change from steady state surface temperatures in excess of $100^{\circ} \mathrm{C}$ to a state below the boiling point of water allowing liquid to infiltrate into the dome interior.

[9] For example, with zero rainfall rate $(r=0)$ and $m_{g}=$ $3.5 \times 10^{-3} \mathrm{~kg} \mathrm{~s}^{-1} \mathrm{~m}^{-2}, T_{s}=210^{\circ} \mathrm{C}$. If $r$ is increased to an unexceptional level of $2.5 \mathrm{~mm} \mathrm{hr}^{-1}, T_{s}$ decreases substantially, to $163^{\circ} \mathrm{C}$. However, this is still well above $100^{\circ} \mathrm{C}$. Hence, all the rainwater that falls onto the dome surface will boil; no infiltration of the dome will occur, and the rainfall is unlikely to trigger any volcanic activity. If $r$ is doubled to $5 \mathrm{~mm} \mathrm{hr}^{-1}, T_{s}$ is now close to $100^{\circ} \mathrm{C}$. Further increases to this modest rainfall rate forces $T_{s}$ below $100^{\circ} \mathrm{C}$. Not all the rainwater would boil at the surface, though there would still be significant evaporation. In this case rainwater could infiltrate into the dome, resulting in new gas-water interactions beyond the scope of the current model. Hence, results are not shown in Figure 1 for $r>r_{c}$ (and $T_{s}<100^{\circ} \mathrm{C}$ ), where

$$
\begin{aligned}
r_{c}= & -\frac{1}{\rho_{l}\left(c_{l} \Delta T+h_{l g}\right)}\left[\frac{m_{g} c_{g}\left(T_{L}-T_{s}\right)}{\left(1-e^{-\beta L}\right)}+\varepsilon \sigma\left(T_{s}^{4}-T_{a}^{4}\right)\right. \\
& \left.+S_{h} \rho_{a} c_{a} u_{a}\left(T_{s}-T_{a}\right)\right],
\end{aligned}
$$

with $T_{s}=100^{\circ} \mathrm{C}(373 \mathrm{~K})$. This critical rainfall rate $r_{c}$ divides the dry dome regime from a possible liquid infiltration regime, and $r_{c}$ increases with magmatic gas flux (Figure 2). For a mass flux $m_{g}=3.5 \times 10^{-3} \mathrm{~kg} \mathrm{~s}^{-1} \mathrm{~m}^{-2}$, we find $r_{c}=$ $6.4 \mathrm{~mm} \mathrm{hr}^{-1}$, with zero wind speed $\left(u_{a}=0\right)$. Such rainfall rates are frequently exceeded at the SHV [Matthews et al., 2002, 2009]. Intense rainfall is usually associated with high wind speeds and large convective heat losses. These act to decrease the critical rainfall rate (Figure 2), raising the probability of significant rainfall-volcanic interactions.

\section{Time-Dependant Rainfall-Induced Cooling}

[10] The results from section 3 imply that the steady state response of a volcanic dome to realistic rainfall rates is to allow rainwater into the dome and so enhance rainwatervolcano interactions. However, if the time taken to reach such steady state solutions is long, compared to the typical life span of the local rain-bearing component of a weather system (a few hours), then rainwater infiltration is unlikely.

[11] The time-dependent cooling of the lava dome is now investigated. First, a relatively low magmatic gas flux of $m_{g}=2 \times 10^{-3} \mathrm{~kg} \mathrm{~s}^{-1} \mathrm{~m}^{-2}$ is chosen, for which the critical rainfall rate is $r_{c}=2.2 \mathrm{~mm} \mathrm{hr}^{-1}$ (Figure 2). The model is initiated with the steady state profile for zero rainfall, corresponding to a surface temperature of $155^{\circ} \mathrm{C}$. A steady rainfall rate is then switched on, and the model is integrated forward in time. The cooling curve for the critical rainfall rate (thick solid line in Figure 3a) shows that this equilibrium would take several hours to approach. However, if $r \geq 5 \mathrm{~mm} \mathrm{hr}^{-1}$, a surface temperature of $100^{\circ} \mathrm{C}$ is reached in $30 \mathrm{~min}$ or less. Sustained rainfall rates of $5 \mathrm{~mm} \mathrm{hr}^{-1}$ at the SHV are frequent (over 200 such independent weather systems in a 3-year period), and these are associated with significantly enhanced volcanic activity in the following few hours [Matthews et al., 2009]. The timescale of cooling during realistic rainfall rates is fast enough for the dome to cool to $100^{\circ} \mathrm{C}$, and subsequent rainwater to infiltrate the dome.

[12] If the magmatic gas flux is increased to $m_{g}=3.5 \times$ $10^{-3} \mathrm{~kg} \mathrm{~s}^{-1} \mathrm{~m}^{-2}$, with zero rainfall, then the steady state surface temperature is higher, at $210^{\circ} \mathrm{C}$. For a fixed rainfall rate, it then takes longer to cool the surface to $100^{\circ} \mathrm{C}$ (Figure 3b). For example, with $r=10 \mathrm{~mm} \mathrm{hr}^{-1}$, it takes 6 minutes when $m_{g}=2 \times 10^{-3} \mathrm{~kg} \mathrm{~s}^{-1} \mathrm{~m}^{-2}$ (Figure 3a), but it takes 40 minutes when $m_{g}=3.5 \times 10^{-3} \mathrm{~kg} \mathrm{~s}^{-1} \mathrm{~m}^{-2}$ (Figure 3b). This is because the higher gas flux advects more heat up to the surface. A larger latent heat flux (and rainfall rate) is then needed to counteract that and subsequently maintain the surface temperature at $100^{\circ} \mathrm{C}$. Hence, more rainwater will evaporate and less is available to infiltrate the dome.

\section{Restricted Gas Flow: Pressure Increase}

[13] Once the surface temperature falls below $100^{\circ} \mathrm{C}$, rainwater will infiltrate the dome and will boil rapidly. This evaporation rapidly cools the upper layer of rock, allowing further infiltration of liquid water. When rainwater occupies some of the void spaces of the matrix, that space is unavailable for magmatic gas to flow upwards, so the effective permeability of the matrix is reduced and the overall magmatic gas flow is restricted.

[14] The depth of lava dome which can be cooled by rainwater evaporation from a storm lasting $\Delta t=100$ minutes, can be estimated by balancing the energy required to evaporate all the water landing on the dome $\left(\Delta t r \rho_{l} h_{l g}\right)$, with the energy provided by thermal diffusion in the solid matrix $\left(L_{\text {rind }} c_{r} \rho_{r} \Delta T\right)$, where the resulting chilling rind thickness $L_{\text {rind }}$, is the depth to which the lava dome is cooled an average amount $\Delta T$ to reach $100^{\circ} \mathrm{C}$. Following (3), the temperature increases rapidly with depth in the thermal boundary layer just below the surface, and therefore we estimate $\Delta T=250^{\circ} \mathrm{C}$. Over a 100 minute rainfall event with a sustained rainfall rate, $r=20 \mathrm{~mm} \mathrm{hr}^{-1}$,

$$
L_{\text {rind }} \sim \frac{\Delta t r h_{l g}}{c_{r} \Delta T} \frac{\rho_{l}}{\rho_{r}}=0.12 \mathrm{~m} .
$$

Notice, this is a dome-wide average chilling rind depth and locally, after the surface has been quenched to the boiling 


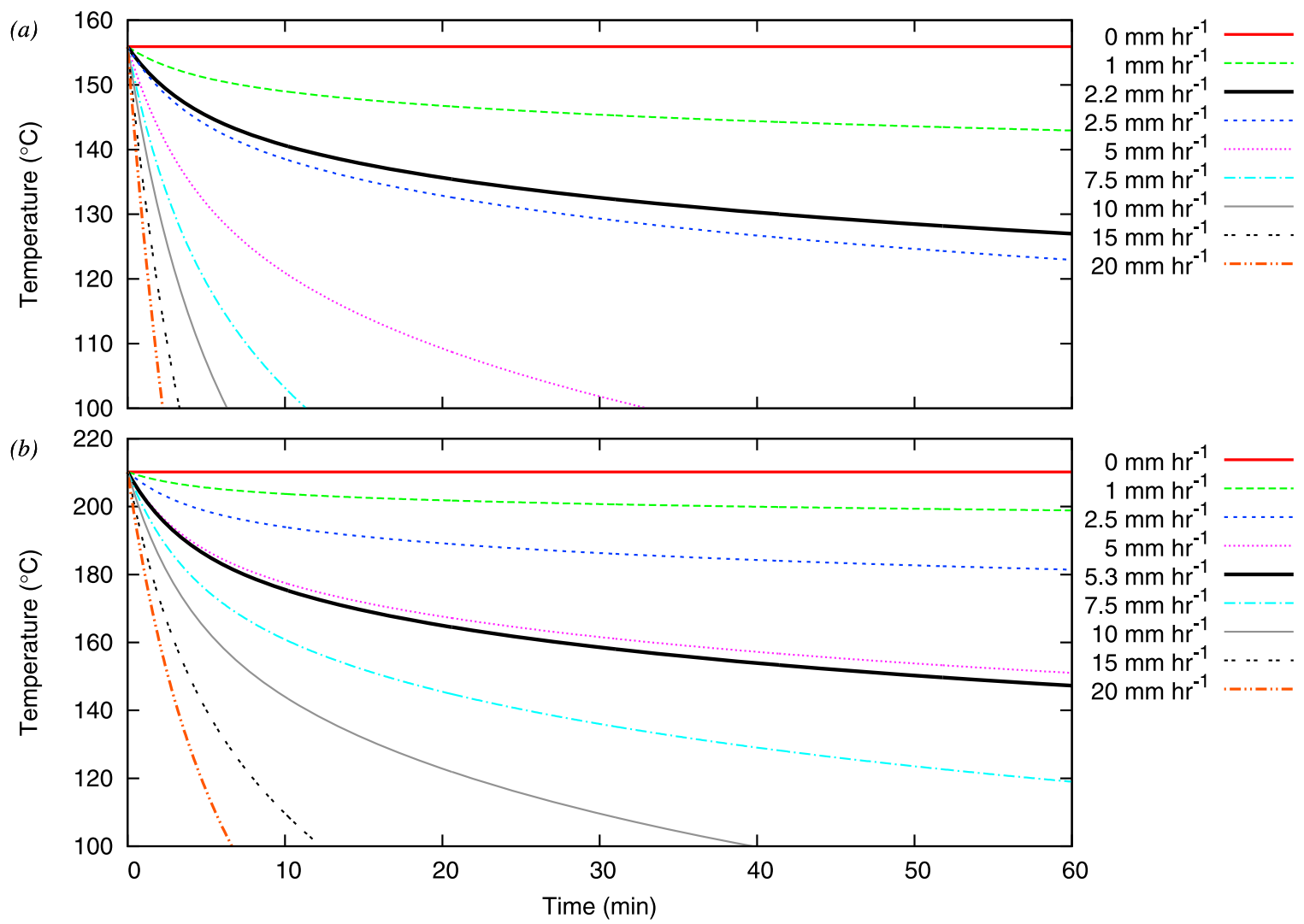

Figure 3. Surface temperature of time-dependent rainfall induced lava dome cooling, starting from a dry steady state with (a) $m_{g}=2 \times 10^{-3}$ and (b) $m_{g}=3.5 \times 10^{-3} \mathrm{~kg} \mathrm{~s}^{-1} \mathrm{~m}^{-2}$, for rainfall rates $r$, between 1 and $20 \mathrm{~mm} \mathrm{hr}^{-1}$ and $u_{a}=5 \mathrm{~m} \mathrm{~s}^{-1}$. The curves for critical rainfall rates are shown by thick solid lines. Note the vertical scale for Figure $3 \mathrm{~b}$ is twice that of Figure $3 \mathrm{a}$.

point, significant amounts of liquid may flow over the surface and inhomogeneously accumulate in some individual cracks and crack networks, resulting in a significantly higher local effective rainfall depth, with a corresponding linear increase in the chilling rind depth. Therefore, given the inhomogeneous nature of the lava dome surface, the local chilling rind depth may be significantly deeper than the dome-wide average, with a depth of approximately $0.5 \mathrm{~m}$ being possible in regions where the features on the dome surface act to locally accumulate four times the average rainfall.

[15] Another estimate for the infiltration depth is given by considering the conservation of rainwater volume. A rainstorm with rainfall rate, $r=20 \mathrm{~mm} \mathrm{hr}^{-1}$ lasting $\Delta t=$ 100 minutes generates an average depth of rainwater, $r \Delta t=$ $0.04 \mathrm{~m}$ over the dome. If all the rainwater were to infiltrate the dome, rather than evaporate, then the amount of rainwater occupying the void-spaces would be given by $\phi s L_{\text {cold }}$, where $L_{\text {cold }}$ is the maximum infiltration depth assuming a uniform saturation, $s$, in a porous medium with porosity, $\phi$. In a completely saturated $(s=1)$ porous medium with $\phi=$ 0.2 , rainwater volume conservation now gives an estimate for the depth of liquid infiltration of

$$
L_{\text {cold }} \sim \frac{\Delta t r}{\phi s}=0.17 \mathrm{~m}
$$

However, if all the void-spaces in a layer near the surface became completely saturated with rainwater, then there would be zero flux of magmatic gas through the saturated region and no magmatic gas could escape the dome. From Darcy's law, completely blocking all the void-spaces to gas flow in this manner is equivalent to applying a (Neumann) boundary condition at the surface in which the pressure gradient is zero. With the application of this type of boundary condition the magmatic gas pressure just below the surface doubles over a time period of less than a minute. If only a proportion of the void-space near the surface is saturated $(0<s<1)$, then magmatic gas can still escape from the dome, and magmatic gas pressure rises are much more gradual. Pressure increases occurring over a time-scale commensurate with observed dome activity, suggest saturations in the range $0.1<s<0.5$. If saturations in this range are uniform across the partially saturated region, then rainfall volume conservation implies a range of maximum infiltration depths in the range $0.4<L_{\text {cold }}<2.0 \mathrm{~m}$. The infiltration depth in a cold porous medium provides a limit on the infiltration depth, as some liquid is evaporated when cooling the initially hot dome. However, in regions where surface features allow the flow of water over the surface prior to infiltrations, an effective local rainfall rate in excess of the dome-wide average is possible with a corresponding increase in infiltration depth.

[16] If the infiltration of rainwater into the dome is driven by gravity, then the speed of liquid descent over a given 
time determines the maximum infiltration depth which can become saturated, $L_{\text {infil }}$, by

$$
L_{\text {infil }} \sim \frac{\Delta t \mathcal{K} \rho_{\ell} g}{\mu_{l}},
$$

where $\mathcal{K}$ is the permeability of the dome and the density and viscosity of the rainwater are denoted $\rho_{l}$ and $\mu_{l}$, respectively. Laboratory measurements of small samples of lava dome give permeabilities in the range $\mathcal{K}=10^{-11}$ to $10^{-15} \mathrm{~m}^{2}$ [Melnik and Sparks, 2002], and when taken in conjunction with a 100 minute rainstorm, this suggests an infiltration depth, $L_{\text {infil }}$, between $0.21 \times 10^{-4}$ and $2.1 \mathrm{~m}$. However, with a permeability as small as $10^{-15} \mathrm{~m}^{2}$, the steady-state magmatic gas pressure gradient necessary to drive the observed gas flux up through the dome induces a pore pressure much greater than the overburden pressure [Hicks et al., 2009]. This discrepancy can be resolved by noting that the measured permeability of laboratory scale samples does not include any of the large features of the dome porous network, such as large cracks and fissures. In these regions the permeability is necessarily much higher, and a permeability (and corresponding liquid infiltration depth) at the larger end of this spectrum is to be expected when considering large scale crack networks close to the dome surface.

[17] Before proceeding to investigate the time-dependent temperature and magmatic gas pressure evolution in the dome, it is worth asking what combination of factors is most likely to contribute to increased pore pressure, potentially causing small Vulcanian eruptions and ultimately lava dome failure? Rather than a uniform, plug like liquid descent, the infiltration depth will depend upon the local cracks, fissures and fumaroles through their associated porosity and permeability, with wide variations in the local infiltration depth across the dome. Some liquid may also flow over the rough dome surface and collect in specific cracks on the surface, leading to a local effective rainfall rate greatly in excess of the dome-wide average. The more rainwater that is available, the greater the potential for the rainwater to restrict the magmatic gas flow. Therefore, locations which have an above average effective rainfall rate will increase the risk factors associated with pore pressure build up. Similarly, locations with large pressure gradients just below the surface reduce the size of the pressure increase required to initiate dome instability. High pressure gradients and large gas flow are often present in cracks and fissures on the dome surface. Further, we can say more about the particular nature of cracks and fissures most at risk: individual crack networks which allow the gas to escape through dry side vents when the main release pathway becomes (partially) blocked by rainwater are able to relieve the pore pressure build up. Conversely, crack networks, which are essentially onedimensional, and do not have side vents (or other features through which the increased gas pressure may be relieved), are likely to generate the highest pressures, and these are the locations most at risk of dangerous pressure build ups.

[18] In areas at greatest risk of dome instability initiation, the energy and mass balances considered suggest a liquid infiltration depth of approximately $0.5 \mathrm{~m}$. In this partially saturated rind, just below the surface, liquid occupies some of the void-spaces and this will constrict the pathways through which magmatic gas can escape. In turn this leads to a pressure increase in the magmatic gas. Rather than model these complex physical processes by explicitly considering the flow of both liquid and gas within the partially saturated region, we assume the liquid is restricted to a narrow region near the surface and model the restriction in the escape of magmatic gas by means of a modified boundary condition applied at the dome surface. Hence, when the surface temperature is cooled to the boiling point of water $\left(T_{s}=100^{\circ} \mathrm{C}\right)$ by the evaporation of rainwater, the boundary conditions described in section 2 are changed. The two time-dependent parabolic partial differential equations corresponding to energy and magmatic gas mass conservation each satisfy one boundary condition at the surface. These are changed to a (Dirichlet) boundary condition $T(0, t)=100^{\circ} \mathrm{C}$, in the energy conservation equation and a (Neumann) boundary condition on the pressure gradient corresponding to the restriction of the volume flow of gas in the gas mass conservation equation. The magmatic gas volume flux at the surface per unit cross section of void-space $\left.v_{g}\right|_{z=0}$, is given by Darcy's law in the form

$$
\left.v_{g}\right|_{z=0}=-\left.\frac{\mathcal{K}}{\phi \mu_{g}} \frac{\partial p_{g}}{\partial z}\right|_{z=0},
$$

where $\mu_{g}$ is the dynamic viscosity of the magmatic gas. The volume flux of magmatic gas through the dome surface when the surface temperature first reaches $100^{\circ} \mathrm{C}$ is denoted $V$. The subsequent changes in effective gas permeability and porosity in the partially saturated region near the surface are assumed to reduce the magmatic gas volume flux through the surface, after infiltration commences, to $\alpha V$, where the constant $\alpha$ is chosen from the interval $0<\alpha<1$.

[19] Notice, the effect of changing the pressure boundary condition from a (Dirichlet) condition on the pressure to a (Neumann) condition on the pressure gradient, creates an apparent pressure discontinuity between the atmospheric pressure and the pressure just below the surface of the dome (see Figure 4). This apparent pressure discontinuity at the surface is the result of effectively shrinking to zero height the partially saturated region. This approximation is justified by the small vertical extent of this region compared to the lava dome carapace thickness. The shrinkage to zero height is done to obtain simply an idealized boundary condition at the surface. If we were to model explicitly the partially saturated region, we would find a pressure gradient there greater than in the dry region below, as the pressure varies continuously between the pressure at the saturation interface and atmospheric pressure. This would induce a faster flow rate, but volume fluxes would remain in the Darcy regime [Hicks, 2008].

[20] To return to the discussion after equation (9), plausible values of $\alpha$ are chosen based on the available volume of rainwater. For example, when forced by the actual rainfall data collected in the runup to the 29 July 2001 dome collapse, the purely diffusive model of lava dome cooling of Matthews and Barclay [2004] predicts an equivalent depth of rainwater of $30 \mathrm{~mm}$ at the time of dome failure. This is the depth of rainwater left over after evaporation to balance the flux of diffusive heat from below. In our current model, more rainwater must be evaporated to cool and maintain the surface temperature at or below $100^{\circ} \mathrm{C}$ because of the additional heat flux from magmatic gas advection. However, as 


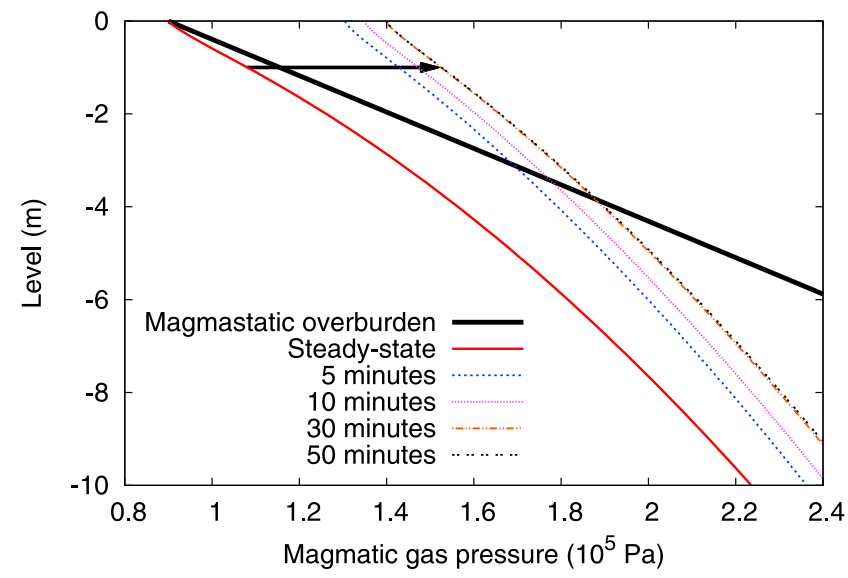

Figure 4. Magmatic gas pressure profiles measured from the time the surface temperature reaches $100^{\circ} \mathrm{C}$ for $m_{g}=$ $3.5 \times 10^{-3} \mathrm{~kg} \mathrm{~s}^{-1} \mathrm{~m}^{-2}, r=10 \mathrm{~mm} \mathrm{hr}^{-1}$ and $u_{a}=5 \mathrm{~m} \mathrm{~s}^{-1}$. Subsequently, the magmatic gas volume flux through the surface is only $\alpha=0.7$ of the value when the surface temperature first reaches $100^{\circ} \mathrm{C}$. The overburden pressure is shown by the thick solid line. the largest component of the heat flux to the surface is thermal diffusion, a significant proportion of this equivalent pool depth will be available to infiltrate the dome. If an equivalent depth of water of $10 \mathrm{~mm}$ uniformly distributes itself over the top $200 \mathrm{~mm}$ of dome, then this corresponds to a reduction of porosity from 0.2 to 0.15 . Costa [2006] fitted the classical Kozeny-Carman porosity-permeability relationship to rock samples from SHV. For small values of the porosity $\mathcal{K} \propto \phi^{2.76}$. This, and (5) give a value for $\alpha$ of approximately 0.6 . Hence, $\alpha$ might vary from nearly one (a small decrease in volume flux) down to approximately 0.5 .

[21] Figure 4 shows the evolution of the pressure profile in a dome when $m_{g}=3.5 \times 10^{-3} \mathrm{~kg} \mathrm{~s}^{-1} \mathrm{~m}^{-2}$, and $\alpha=0.7$. Over the 50 minutes after the onset of liquid infiltration, the pressure profile tends to a new steady state in which the pressure immediately below the surface is $1.4 \times 10^{5} \mathrm{~Pa}$, a $60 \%$ increase on the surface pressure prior to the restriction of the magmatic gas volume flux. This increased magmatic gas pressure exceeds the overburden pressure, from the weight of overlying rock, in the top $4 \mathrm{~m}$ of the dome. If the dome is close to an unstable configuration, then this extra pressure perturbation may be sufficient to start a dome collapse.

[22] Figure 5 shows the increase of magmatic gas pressure immediately below the surface and at a depth of $5 \mathrm{~m}$, for

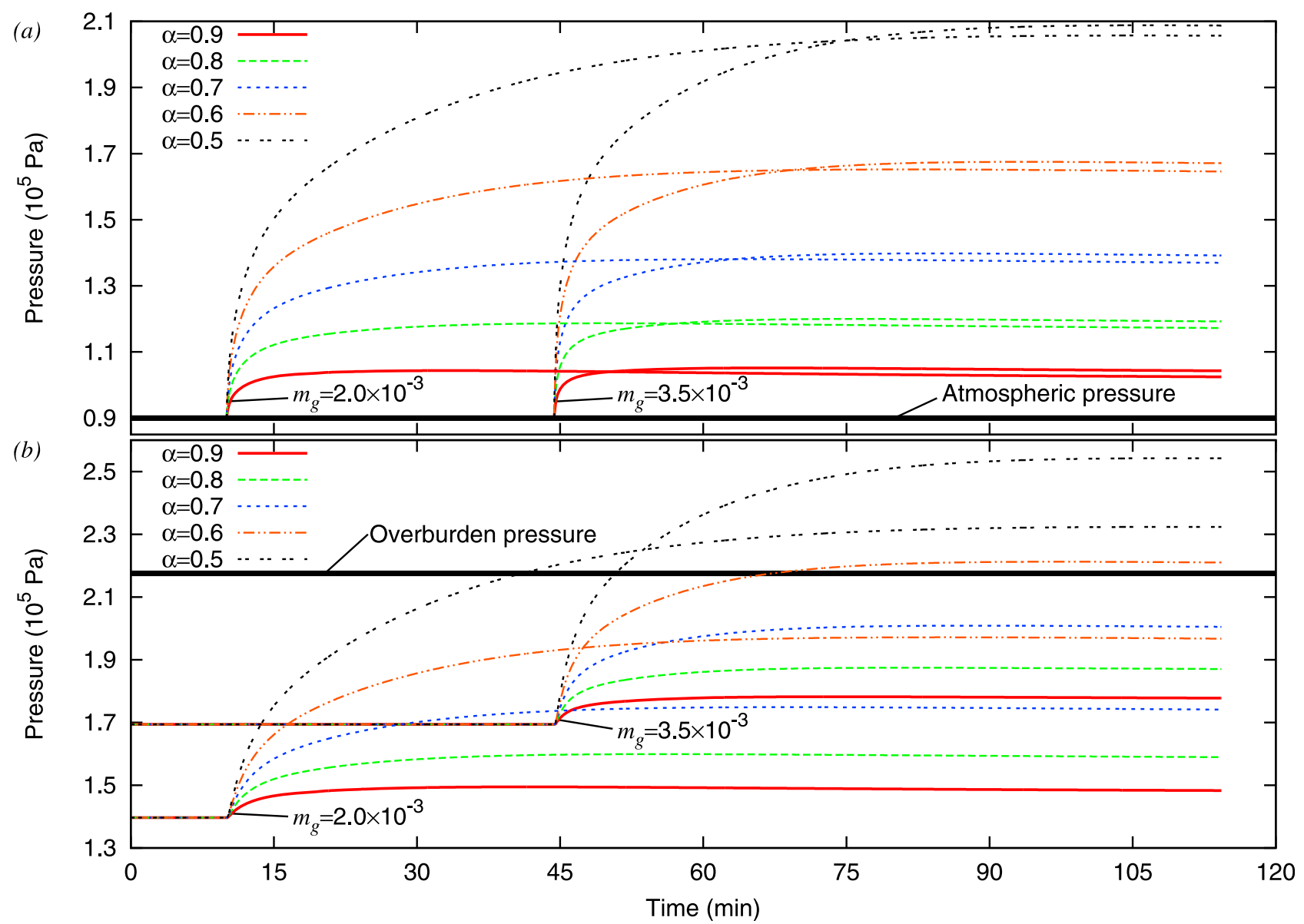

Figure 5. Pressure evolution (a) immediately below and (b) $5 \mathrm{~m}$ below the dome surface. Rainfall starts at $t=0$ with an intensity of $10 \mathrm{~mm} \mathrm{hr}^{-1}$, and $u_{a}=5 \mathrm{~m} \mathrm{~s}^{-1}$. The pressure evolution (starting from the steady state profiles) corresponding to $m_{g}=2 \times 10^{-3}$ and $3.5 \times 10^{-3} \mathrm{~kg} \mathrm{~s}^{-1} \mathrm{~m}^{-2}$ are shown for $\alpha=0.5,0.6,0.7$, 0.8 and 0.9 . The overburden pressure is shown by the thick horizontal line in Figure $5 \mathrm{~b}$. 
different levels of flow restriction $\alpha$, and for $m_{g}=2 \times 10^{-3}$ and $3.5 \times 10^{-3} \mathrm{~kg} \mathrm{~s}^{-1} \mathrm{~m}^{-2}$. The pressure rises more rapidly in the cooler dome (with the lower mass flux), and the pressure increase is largest when the volume flux is most restricted. The gas pressure is greater when $m_{g}$ is higher. This means less pressure increase is required to reach the overburden pressure (a criterion for dome collapse), even though more cooling time is required before the volume flux becomes restricted. If $\alpha=0.6$, the magmatic gas pressure in a region where $m_{g}=3.5 \times 10^{-3} \mathrm{~kg} \mathrm{~s}^{-1} \mathrm{~m}^{-2}$, reaches the overburden pressure 70 minutes after the onset of rainfall, but in regions of lower mass flux (e.g., $m_{g}=2 \times 10^{-3} \mathrm{~kg} \mathrm{~s}^{-1} \mathrm{~m}^{-2}$ ) the pressure may never reach the overburden pressure.

\section{Conclusions}

[23] Rainfall can cool the surface of a lava dome to below the boiling point, so rainwater can then infiltrate the dome, where its subsequent interactions with the hot rock may lead to the collapse of the dome. The critical rainfall rate required to cool the lava dome surface to the boiling point of water has been determined as a function of the magmatic gas mass flux through the dome. The surface temperature is greatest where the magmatic gas flux is highest, and these regions of the dome surface require more time and the greatest evaporation of rainfall to cool. Once the surface temperature is below the boiling point, rainwater can infiltrate the dome, and an idealized model for the blocking based on the restriction of the magmatic gas volume flux is used. For realistic parameter values, this predicts an increase in the magmatic gas pressure of $60 \%$ immediately below the dome surface over a period of one hour after the onset of liquid infiltration. These gas pressures are in excess of the overburden pressures in the top few meters, potentially leading to the collapse of the dome. The scale of the reduction in volume flux at the surface by the infiltration of rainwater has been justified by mass and energy conservation balances and on observational grounds.

[24] The time from the start of rainfall to the start of dome failure is about one hour, which accords with observations reported by Matthews et al. [2009]. They also report that this was followed over the next few hours by a deeper response, with an increase in subsurface long-period rockfalls (from shallow degassing), then long-period and hybrid activity (source depth approximately $1 \mathrm{~km}$ ).

[25] The lava dome is most at risk of increased subsurface gas pressures in regions with high porosity and permeability. On a dome these high porosity regions will correspond to crack networks, fissures and fumaroles which are visible covering the surface. Once liquid infiltrates these cracks, the gas pressure build up is most rapid in regions with above average magmatic gas fluxes. However, there is a trade-off required as regions with large magmatic gas fluxes are the hottest, and therefore require most time and rainwater evaporation to cool. Local crack networks and fissures, which allow gas to escape through dry side vents also have a reduced potential to cause subsurface magmatic gas pressure build up. Conversely those crack networks and fissures which are essentially one dimensional and do not have branching networks of gas pathways close to the surface provide the best conditions for a build up of gas pressure. The first effects of increased pressure in the crack networks of a dome will be felt round the periphery of the dome, where the surface slope of the dome acts to reduce the effective overburden pressure. However, we have shown that pressures can eventually approach the overburden pressure in crack networks situated even in a region where the dome surface is flat.

[26] Future work will concentrate on explicitly modeling the liquid descent into the upper layers of the dome, its staunching of the upward flow of magmatic gas, and the consequent increase in internal pore pressure. The extension to two and three spatial dimensions would allow the pressure to be calculated in crack networks from which gas is able to escape though dry side vents. However, such results would be limited by the quality of data on spatial variations in porosity across different types of dome features.

[27] Operationally, our theory suggests that combined monitoring of current magmatic gas fluxes and predicted rainfall rates would be useful inputs, along with an overall assessment of dome stability, to volcanic hazard management.

\section{Appendix A: Model Equations}

[28] The conservation of mass, momentum and energy in a porous lava dome carapace is governed by an extension of the model of Hicks et al. [2009]. In the dome carapace, the solid matrix is assumed to be rigid with magmatic gases flowing through the void-spaces in the rock. In one spatial dimension $z$, aligned parallel to the gas pressure gradient and positive in the upwards direction, conservation of magmatic gas mass is governed by

$$
\frac{\partial \rho_{g}}{\partial t}+\frac{\partial}{\partial z}\left(\rho_{g} v_{g}\right)=0
$$

where the magmatic gas density is denoted $\rho_{g}$, and the volume flux of magmatic gas per unit cross-sectional area of voidspace is denoted $v_{g}$. The magmatic gas momentum conservation is assumed to be governed by Darcy's law and has the form

$$
v_{g}=-\frac{\mathcal{K}}{\phi \mu_{g}} \frac{\partial p_{g}}{\partial z}
$$

where magmatic gas volume flux per unit cross-sectional area of void-space is proportional to the pressure gradient, with a constant of proportionality involving the permeability $\mathcal{K}$, the porosity $\phi$ and the magmatic gas viscosity $\mu_{g}$. Notice, the volume flux of magmatic gas per unit surface area of dome is given by $\phi v_{g}$. For the range of pressure gradients commonly found in a lava dome the effect of gravity on the flow is negligible.

[29] Local thermal equilibrium exists between the magmatic gas and solid dome where condition (1) is satisfied. This condition holds in the high-porosity regions of interest. Therefore, the evolution of the common magmatic gas and rock temperature $T$, is governed by the conservation of energy equation

$$
\frac{\partial}{\partial t}\left[\left\{(1-\phi) c_{r} \rho_{r}+\phi c_{g} \rho_{g}\right\} T\right]+\frac{\partial}{\partial z}\left(\phi c_{g} \rho_{g} v_{g} T\right)-k_{e} \frac{\partial^{2} T}{\partial z^{2}}=0
$$


where $c_{r}$ and $c_{g}$ and the specific heat capacities of the rock and the magmatic gas, respectively, and $k_{e}$ is the effective thermal conductivity of the (gas filled) porous medium.

[30] The model is completed by an equation of state for the magmatic gas. We assume the magmatic gas behaves as an ideal gas with $p_{g}=\rho_{g} R_{g} T$, where (due to the magmatic gas composition), the ideal gas constant $R_{g}$ for water vapor is used. In this simplified model liquid rainwater is not present explicitly in these conservation laws. Its presence is assumed to be restricted to a nominal layer near the surface, with thickness much smaller than the typical thickness of the carapace region and modeled using the modified boundary conditions at the surface, as described in section 5 .

[31] Acknowledgments. We thank Jenni Barclay and Richard Herd for useful discussions and three anonymous reviewers whose comments helped to significantly improve the manuscript.

\section{References}

Barclay, J., J. E. Johnstone, and A. J. Matthews (2006), Meteorological monitoring of an active volcano: Implications for eruptive prediction, J. Volcanol. Geotherm. Res., 150, 339-358, doi:10.1016/j. jvolgeores.2005.07.020.

Carn, S. A., R. B. Watts, G. Thompson, and G. E. Norton (2004), Anatomy of a lava dome collapse: The 20 March 2000 event at Soufrière Hills Volcano, Montserrat, J. Volcanol. Geotherm. Res., 131, 241-264, doi:10.1016/S0377-0273(03)00364-0.

Costa, A. (2006), Permeability-porosity relationship: A reexamination of the Kozeny-Carman equation based on a fractal pore-space geometry assumption, Geophys. Res. Lett., 33, L02318, doi:10.1029/2005GL025134.

Edmonds, M., D. Pyle, and C. Oppenheimer (2001), A model for degassing at the Soufrère Hills Volcano, Montserrat, West Indies, based on geochemical data, Earth Planet. Sci. Lett., 186, 159-173, doi:10.1016/ S0012-821X(01)00242-4.

Elsworth, D., B. Voight, G. Thompson, and S. R. Young (2004), Thermalhydrologic mechanism for rainfall-triggered collapse of lava domes, Geology, 32(11), 969-972.

Hicks, P. (2008), A thermodynamic model of rainwater and magmatic vapour flow in a porous medium: Triggering of a volcanic lava dome collapse, Ph.D. thesis, School of Math., Univ. of East Anglia, Norwich, U. K.

Hicks, P. D., A. J. Matthews, and M. J. Cooker (2009), The thermal structure of a gas-permeable lava dome and timescale separation in its response to perturbation, J. Geophys. Res., 114, B07201, doi:10.1029/ 2008JB006198.

Hort, M., R. Seyfried, and M. Voge (2003), Radar Doppler velocimetry of volcanic eruptions: Theoretical considerations and quantitative documentation of changes in eruptive behaviour at Stromboli volcano, Italy, Geophys. J. Int., 154, 515-532, doi:10.1046/j.1365-246X.2003.01982.x.
Kaviany, M. (1995), Principles of Heat Transfer in Porous Media, Springer, New York.

Mastin, L. G. (1994), Explosive tephra emissions at Mount St. Helens, 1989-1991: The violent escape of magmatic gas following storms?, Geol. Soc. Am. Bull., 106, 175-185.

Matthews, A. J., and J. Barclay (2004), A thermodynamical model for rainfall-triggered volcanic dome collapse, Geophys. Res. Lett., 31, L05614, doi:10.1029/2003GL019310.

Matthews, A. J., J. Barclay, S. A. Carn, G. Thompson, J. Alexander, R. A. Herd, and C. Williams (2002), Rainfall-induced volcanic activity on Montserrat, Geophys. Res. Lett., 29(13), 1644, doi:10.1029/ 2002GL014863.

Matthews, A. J., J. Barclay, and J. E. Johnstone (2009), The fast response of volcano-seismic activity to intense precipitation: Triggering of primary volcanic activity by rainfall at Soufriére Hills Volcano, Montserrat, J. Volcanol. Geotherm. Res., 184(3-4), 405-415, doi:10.1016/j.jvolgeores.2009.05.010

Melnik, O., and R. S. J. Sparks (2002), Dynamics of magma ascent and extrusion at Soufrière Hills Volcano, Montserrat, in The Eruption of Soufrière Hills Volcano, Montserrat, From 1995 to 1999, Memoirs, vol. 21, edited by T. Druitt and B. Kokelaar, pp. 115-152, Geol. Soc. of London, London.

Neuberg, J. (2000), External modulation of volcanic activity, Geophys. J. Int., 142, 232-240, doi:10.1046/j.1365-246x.2000.00161.x.

Oppenheimer, C., P. W. Francis, D. A. Rothery, and R. W. T. Carlton (1993), Infrared image analysis of volcanic thermal features: Lascar volcano, Chile 1984-1992, J. Geophys. Res., 98, 4269-4286.

Simmons, J., D. Elsworth, and B. Voight (2004), Instability of exogenous lava lobes during intense rainfall, Bull. Volcanol., 66(8), 725-734, doi:10.1007/s00445-004-0353-y.

Taron, J., D. Elsworth, G. Thompson, and B. Voight (2007), Mechanisms for rainfall-concurrent lava dome collapses at Soufrière Hills Volcano, 2000-2002, J. Volcanol. Geotherm. Res., 160, 195-209, doi:10.1016/j. jvolgeores.2006.10.003

Violette, S., G. de Masily, J. P. Carbonnel, P. Goblet, E. Ledoux, S. M. Tijani, and G. Vouille (2001), Can rainfall trigger volcanic eruptions? A mechanical stress model of an active volcano: 'Piton de la Fournaise', Reunion Island, Terra Nova, 13, 18-24.

Voight, B., E. K. Constantine, S. Siswowidjoyo, and R. Torley (2000), Historical eruptions of Merapi Volcano, central Java, Indonesia, 1768-1998, J. Volcanol. Geotherm. Res., 100, 69-138, doi:10.1016 S0377-0273(00)00134-7.

Yamasato, H., S. Kitagawa, and M. Komiya (1998), Effect of rainfall on dacitic lava dome collapse at Unzen Volcano, Japan, Pap. Meteorol. Geophys., 48(3), 73-78, doi:10.2467/mripapers.48.73.

M. J. Cooker, School of Mathematics, University of East Anglia, Norwich NR4 7TJ, UK. (m.cooker@uea.ac.uk)

P. D. Hicks, Department of Mathematics, University College London, Gower Street, London WC1E 6BT, UK. (p.hicks@ucl.ac.uk)

A. J. Matthews, School of Environmental Sciences, University of East Anglia, Norwich NR4 7TJ, UK. (a.j.matthews@uea.ac.uk) 